\title{
Копирующий пневмопривод
}

\author{
(C) В.Н. Пильгунов, К.Д. Ефремова
}

МГТУ им. Н.Э. Баумана, Москва, 105005, Россия

Предложено схемное решение копирующего пневмопривода с двумя дифференциальными пневмоцилиндрами, один из которых выполняет функиию задающего пневмочилиндра, а другой - копирующего. Разработана математическая модель копирующего пневмопривода и проверена ее адекватность.

Ключевые слова: копирующий пневмопривод, задающий пневмоцилиндр, копирующиий пневмоцилиндр, показатель критичности, параметр истечения, подпиточный клапан, клапан подпора.

Классическая схема пневмопривода возвратно-поступательного движения предусматривает наличие энергетической (компрессора и системы подготовки сжатого воздуха), исполнительной (пневмоцилиндра) и управляющей (пневмораспределителя и аппаратуры управления потоком сжатого воздуха) подсистем. В копирующем пневмоприводе (рисунок) функцию энергетической подсистемы выполняет задающий пневмоцилиндр, приводимый в движение человеком-оператором; управляющая подсистема отсутствует, компрессор работает периодически и поддерживает заданное давление подпитки в ресивере.

Управляющая сила $R_{1}$ на штоке задающего пневмоцилиндра ПЦ1 обеспечивает прямой ход $x_{1}$ поршня, при этом из штоковой полости $S_{b}$ воздух вытесняется в поршневую полость $S_{c}$ копирующего пневмоцилиндра ПЦ2: его шток выдвигается, преодолевая нагружающую силу $R_{2}$. Сжатый воздух, вытесняемый из штоковой полости $S_{d}$ пневмоцилиндра ПЦ2, поступает в поршневую полость $S_{a}$ пневмоцилиндра ПЦ1. При обратном ходе поршня пневмоцилиндра ПЦ1 процесс аналогичен. Таким образом, поршень копирующего пневмоцилиндра следует за движением поршня задающего пневмоцилиндра и выполняет процедуру слежения по положению. Дифференциальность пневмоцилиндров определяет разность объема воздуха, вытесняемого из полости $S_{d}$, и объема воздуха, высвобождаемого полостью $S_{a}$. Это вызывает понижение давления в полости $S_{a}$, срабатывание подпиточного клапана $K_{a}$ и поступление необходимого объема воздуха $V_{0}$ в полость $S_{a}$ из ресивера. Давление в ресивере поддерживается на заданном уровне редукционным клапаном. Одновременно воздух, вытесняемый из штоковой полости $S_{b}$ пневмоцилиндра ПЦ1, поступает в поршневую полость $S_{c}$ пневмоцилиндра 
ПЦ2 и обеспечивает прямой ход $x_{2}$ его поршня с преодолением встречной силы $R_{2}$. При смене знака силы $R_{1}$ поршень пневмоцилиндра ПЦ1 совершает обратный ход, вытесняемые из поршневых полостей объемы воздуха превышают высвобождаемые объемы воздуха из штоковых полостей $S_{b}$ и $S_{d}$, что вызывает срабатывание клапанов подпора $K_{b}$ и $K_{d}$. Поршень пневмоцилиндра ПЦ2 перемещается на величину $x_{2}$, определяемую тянущей силой $R_{2}$. Если сила $R_{2}$ сохраняет знак нагрузки на прямом ходе, то она способствует уборке штока пневмоцилиндра ПЦ2. Давление настройки клапанов подпора должно быть несколько большим, чем давление подпитки $p_{0}$, в противном случае через них будет стравливаться сжатый воздух.

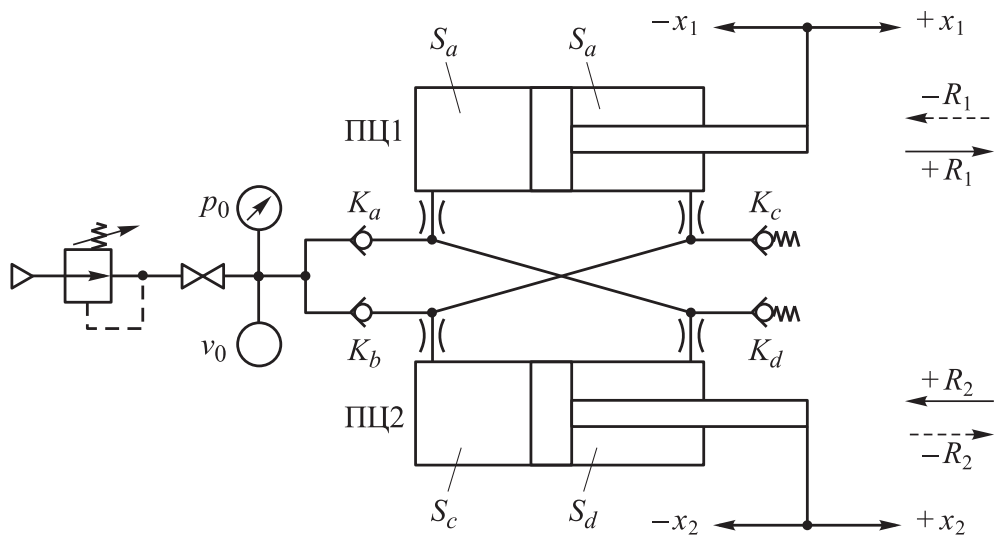

Принципиальная схема копирующего пневмопривода

Термодинамический процесс изменения состояния воздуха может быть описан уравнением

$$
d q=d e+d a,
$$

где $d q-$ удельная теплота, подводимая к воздуху массой $m, d q=d Q / m$; $d e$ - удельное изменение внутренней энергии воздуха объемом $V$, $d e=d E / m ; d a$ - удельная внешняя работа, совершаемая воздухом массой $m$ при его расширении, $d a=d A / m[1,2]$. Копирующий пневмоцилиндр совершает работу $d A>0$ по преодолению знакопеременной силы на штоке. Поршень задающего пневмоцилиндра, выполняющего функцию компрессора, использует подводимую человеком-оператором механическую энергию, в этом случае $d A>0$ и $d a=d e-d q$.

Работа копирующего пневмопривода в зависимости от скорости движения штоков пневмоцилиндров может происходить при различных процессах изменения состояния воздуха. 
В изотермическом процессе подводимая теплота в условиях постоянства температуры $d T=0$ приводит к увеличению объема воздуха $V$ и поршень совершает полезную работу $d A>0$ по преодолению силы $R$ на штоке на пути $d x$. Если рассматривать воздух как идеальный газ, то процесс будет происходить без изменения его внутренней энергии: $d e=0$ и $d q=d a=p d V_{m}=p A_{\text {п }} d x / m$, где $A_{\text {п }}$ - площадь поршня, м²; $V_{m}$ - удельный объем воздуха $V$, отнесенный к его массе $m$ при плотности $\rho, V_{m}=V / m=1 / \rho, \mathrm{M}^{3} / \kappa^{1}$. Учитывая уравнение Менделеева - Клапейрона

$$
p V_{m}=\frac{p}{\rho}=R T,
$$

где $R$ - газовая постоянная воздуха, $R=287$ Дж/(кГ $\left.{ }^{1} \cdot \mathrm{K}^{1}\right)$, определяем удельную работу $a=R T \ln \left(V_{m_{2}} / V_{m_{1}}\right)$. В этом случае уравнение состояния воздуха (1) принимает вид

$$
p_{1} V_{m 1}=p_{2} V_{m 2}=\text { inv, }
$$

или с учетом $V_{m}=1 / \rho$

$$
p_{1} / \rho_{1}=p_{2} / \rho_{2}=\text { inv. }
$$

Изотермический процесс возможен в условиях активного теплообмена пневмоцилиндров с окружающей средой, или при малой скорости движения их поршней $d x / d t=0$.

Изобарический процесс происходит при постоянной силе: $R=$ inv. Если рассматривать воздух как реальный газ, то $d q=C_{p} d T, d e=C_{V} d T$, где $C_{p}$ и $C_{V}$ - удельные теплоемкости воздуха, полученные при его нагреве в условиях постоянства давления $d p=0$ и объема $d V=0$. Уравнение состояния газа (1) имеет вид $\left(C_{p}-C_{V}\right) d T=p d V_{m}=p A_{\text {пा }} d x / d t$.

Газовая постоянная $R$ находится как разность удельных теплоемкостей $R=C_{p}-C_{V}$. Тогда уравнение (1) принимает вид $d a=p A_{\text {п }} d x / d t=R d T$. Интегрирование этого уравнения в пределах изменения температуры $T_{1} \ldots T_{2}$ определяет физическую сущность газовой постоянной

$$
R=\frac{a}{T_{2}-T_{1}}
$$

Адиабатический процесс происходит без подвода теплоты $(d q=0)$, с учетом этого уравнение состояния (1) принимает вид $d e=-d a$, что соответствует равенству $p A_{\Pi} d x / m=-C_{V} d T$. 
Дифференцирование уравнения (2) обусловливает изменение температуры

$$
\frac{d\left(p V_{m}\right)}{R}=\frac{\left(p d V_{m}+V_{m} d p\right)}{R}
$$

Используя показатель адиабаты $k=C_{p} / C_{V}$, получаем

$$
\frac{k d V_{m}}{V_{m}}+\frac{d p}{p}=0
$$

Интегрирование этого равенства позволяет записать уравнение состояния воздуха в адиабатическом процессе: $p_{1} V_{m 1}^{k}=p_{2} V_{m 2}^{k}=$ inv.

Политропический процесс - промежуточный (реальный) процесс между изотермическим $(d q \neq 0)$ и адиабатическим $(d q=0)$ процессами. Вместо показателя адиабаты вводится показатель политропы $n=\left(C_{p}-C_{n}\right) /\left(C_{V}-C_{n}\right)$, который учитывает удельную теплоемкость $C_{n}$ в политропическом процессе. Уравнение состояния воздуха в адиабатическом процессе имеет вид

$$
p_{1} V_{m 1}^{n}=p_{2} V_{m 2}^{n}=\text { inv. }
$$

В термодинамическом процессе, связанном с тепломассообменом, переменность массы воздуха в полостях пневмоцилиндров при движении поршня вызывает нестационарность процесса изменения состояния воздуха и уравнение баланса энергий (1) становится развернутым:

$$
d q_{m}=d q+q_{1} \frac{d m_{1}}{m}=d e+d a+q_{2} \frac{d m_{2}}{m}
$$

где $q_{1}, q_{2}$ - удельная теплота входящего в объем воздуха массой $d m_{1}$ и выходящего из этого объема воздуха массой $d m_{2} ; d q_{m}$ - приращение удельной теплоты в процессе тепломассообмена; $d a-$ совершенная поршнем внешняя удельная работа, $d a=p d V_{m} ; d V_{m}$ - изменение удельного объема, $d V_{m}=d(V / m)=A_{\text {п }} d x / m-V_{0} d_{m} / m$ ( $V_{0}$ - начальный объем полости пневмоцилиндра); $d m$ - приращение массы воздуха в полости пневмоцилиндра, $d m=d m_{1}-d m_{2}$.

В рассматриваемом случае удельная работа составляет

$$
d a=p A_{\Pi} \frac{d x}{m}-p V_{m} \frac{d m}{m} .
$$


В работе [3] приведено выражение для оценки величины $d q_{m}=d q+$ $+\left(I_{1}-k e\right) d m_{1} / m$, где $I_{1}-$ удельное теплосодержание (энтальпия) входящего воздуха массой $d m_{1}, I_{1}=e+p V_{m}$.

С учетом выражений $d e=C_{V} d T ; k=C_{p} / C_{V} ; R=C_{p}-C_{V} ; k-1=R / C_{V}$; $R=C_{V} /(k-1) ; p V_{m}=R T$ находим удельную работу в процессе тепломассобмена:

$$
d a=\frac{p A_{\mathrm{n}} d x}{m}-\frac{e(k-1) d m}{m} .
$$

Введем безразмерный параметр $q_{a}=d q_{m} / m$, оценивающий интенсивность тепломассобмена $d q_{m}$ по сравнению с совершенной внешней работой $d a$ :

$$
q_{a}=\frac{d q+q(I-k e) d m_{1} / m}{p A_{\mathrm{n}} d x / m-e(k-1) d m / m} .
$$

Массовый расход воздуха $G=d m / d t$. После преобразований уравнение (4) принимает вид

$$
q_{a}=\frac{d Q / d t+\left(I_{1}-k e\right) G_{1}}{p A_{\text {п }} d x / d t-e(k-1)\left(G_{1}-G_{2}\right)} .
$$

В выражении (5) слагаемое $p A_{\text {п }} d x / d t$ соответствует мощности термодинамического процесса тепломассообмена, а слагаемое $d Q / d t-$ секундному изменению подводимой теплоты.

Запишем приведенные формулы (1)-(5) для процессов заполнения и опорожнения полостей пневмоцилиндров ПЦ1 и ПЦ2 копирующего пневмопривода.

1. Быстрое опорожнение полости пневмоцилиндра вытеснителем без теплообмена. В этом случае

$$
G_{1}=0 ; d x<0 ; d Q=0 ; q_{a}=0 ; n=k-q_{a}(k-1)=1,4 \text {. }
$$

\section{2. Медленное опорожнение полости пневмоцилиндра в услови-} ях теплообмена. При медленном опорожнении составляющие уравнения (5) принимают следующие значения: $G_{1}=0 ; d x<0 ; d Q<0$;

$$
q_{a}=\frac{d Q / d t}{p A_{\mathrm{n}} d x / d t+e(k-1) G_{2}},
$$


а в условиях изотермического процесса интенсивность теплообмена $d q_{m_{1}}$ равна совершенной работе $q_{a}=1, n=k-q_{a}(k-1)=1$.

3. Заполнение полости пневмоцилиндра переменного объема без совершения внешней работы в условиях отсутствия теплообмена. Этот режим происходит при параметрах процесса $G_{2}=0 ; d x>0 ; p=0$; $d Q=0 ; q_{a}=-\frac{\left(I_{1} / e-k\right)}{k-1}$.

4. Заполнение полости пневмоцилиндра переменного объема с совершением внешней работы по преодолению постоянной силы в условиях отсутствия теплообмена. В этом случае составляющие уравнения (5) имеют следующие значения: $G_{2}=0 ; d x>0 ; p=p_{0}=$ inv; $d Q=0 ; \quad q_{a}=\frac{\left(I_{1} / e-k\right) G_{1}}{p A_{\Pi} d x /(e d t)-(k-1) G_{1}}$

Постоянство давления ( $p=$ inv) соответствует изобарическому процессу $(d p=0)$ и значению безразмерного параметра $q_{a}=3,5$. Совершаемая поршнем внешняя работа будет однозначно связана с массовым расходом $G_{1}$ воздуха, заполняющего полость, и его начальными параметрами (энтальпией $I_{1}$ и внутренней энергией $e$ ).

5. Заполнение полости пневмоцилиндра переменного объема с совершением внешней работы по преодолению позиционной нагрузки без теплообмена. Этот режим характеризуется следующими параметрами процесса: $G_{2}=0 ; d x>0 ; p=p(x) ; d Q=0$; $q_{a}=\frac{\left(I_{1} / e-k\right) G_{1}}{p(x) A_{\Pi} d x /(e d t)-(k-1) G_{1}}$.

Рассмотрим силовые характеристики пневмоцилиндров ПЦ1 и ПЦ2 без учета сил трения в уплотнениях и направляющих с переменными силами на штоках $R_{1}=R_{1}\left(x_{1}\right)$ - управляющая сила, $R_{2}=R_{2}\left(x_{2}\right)$ - нагружающая сила [4]. Необходимо отметить, что в зависимости от режима работы копирующего пневмоцилиндра сила $R_{2}$ может быть как знакопеременной, так и знакопостоянной.

Для задающего пневмоцилиндра ПЦ1:

прямой ход

$$
\left(x_{1}>0\right) p_{a} S_{a}+k_{1} x_{1}-p_{b} S_{b}=0
$$

обратный ход

$$
\left(x_{1}<0\right) p_{b} S_{b}-k_{1} x_{1}-p_{a} S_{a}=0 .
$$


Для копирующего пневмоцилиндра ПЦ2 при знакопостоянной нагрузке $R_{2}$

прямой ход

$$
\left(x_{2}>0\right) p_{c} S_{c}-k_{2} x_{2}-p_{b} S_{b}=0
$$

обратный ход

$$
\left(x_{2}<0\right) p_{d} S_{d}+k_{2} x_{2}-p_{c} S_{c}=0 .
$$

Для копирующего пневмоцилиндра ПЦ2 при знакопостоянной нагрузке $R_{2}$ :

прямой ход

$$
\left(x_{2}>0\right) p_{c} S_{c}-k_{2} x_{2}-p_{d} S_{d}=0
$$

обратный ход

$$
\left(x_{2}<0\right) p_{d} S_{d}-k_{2} x_{2}-p_{c} S_{c}=0 .
$$

Истечение воздуха через подводящие и отводящие устройства пневмоцилиндров происходит с потерями гидравлической энергии. Потери давления на входе в полость пневмоцилиндра будем рассматривать как потери выхода из трубопровода с коэффициентом сопротивления $\xi_{\text {вых }}=1$, а потери давления на выходе из полости пневмоцилиндра - как потери входа в трубопровод с коэффициентом сопротивления $\xi_{\mathrm{Bx}}=0,5$. Это соответствует коэффициентам расхода $\mu_{\mathrm{Bx}}=0,50$ и $\mu_{\text {вых }}=0,82$ на входе в полость пневмоцилиндра и выходе из нее. Значения массовых расходов воздуха через входное и выходное устройства будем рассчитывать по формулам, полученным из уравнения Сен-Венана - Ванцеля с учетом уравнения состояния газа Менделеева - Клапейрона $(\rho=p / R T)$ и соотношения плотностей в политропическом процессе

$$
\rho_{2}=\rho_{1}\left(\frac{p_{2}}{p_{1}}\right)^{1 / n} \text {, или } \rho_{2}=\rho_{1} \beta^{1 / n},
$$

где $\beta$ - относительное (безразмерное) давление, которое будем рассматривать как показатель критичности процесса истечения воздуха под абсолютным давлением $p_{1}$ в газовую среду с абсолютным давлением $p_{2}$ (значения $\beta_{\text {кр }}=0,528 ; \beta<0,528 ; 0,528<\beta<0,83 ; \beta>0,83$ определяют режимы критического, надкритического, докритического истечения воздуха и режим несжимаемой капельной жидкости соответственно [5]), $\beta=p_{2} / p_{1}$. Тогда 


$$
G=\mu A_{\text {п.с }} p_{1}\left[\frac{2 n}{R T_{1}(n-1)}\right]\left(\beta^{2 / n}-\beta^{n+1 / n}\right)^{0,5},
$$

где $A_{\text {п.с }}$ - площадь проходного сечения.

Введем проводимость подводящего (отводящего) устройства пневмоцилиндра $\left(Z=\mu A_{\text {п.с }}\right)$ и параметр истечения воздуха через подводящее (отводящее) устройство

$$
k_{\beta}=\left[\frac{2 n}{R T_{1}(n-1)}\right]\left(\beta^{2 / n}-\beta^{n+1 / n}\right)^{0,5} .
$$

Параметр истечения $k_{\beta}=k_{\beta}(\beta, R, T)$ учитывает свойства газа $(R)$, его абсолютную температуру $(T)$ на входе в устройство, характер процесса истечения (показатель политропы $n$ ) и показатель критичности $(\beta)$. Зависимость параметра истечения от показателя политропы представлена в работе [6]. После подстановки проводимости $Z$ и параметра истечения $k_{\beta}$ в уравнение (12) получим уравнение для определения массового расхода:

$$
G=Z k_{\beta} p_{1}
$$

Исследуем работу копирующего пневмопривода на прямом и обратном ходах поршня задающего пневмоцилиндра ПЦ1.

Прямой ход поршня пневмоцилиндра ПЦ1. Запишем приведенные формулы (6)-(12) для процессов заполнения и опорожнения полостей пневмоцилиндров ПЦ1 и ПЦ2 копирующего пневмопривода.

1. Опорожнение штоковой полости $\boldsymbol{S}_{b}$. Масса воздуха, вытесняемая поршнем из полости $S_{b}$,

$$
d m_{b}=d\left(V_{b} \rho_{b}\right)=S_{b} d\left(x_{1} \rho_{b}\right)=S_{b} x_{10} d \rho_{\mathrm{b}}+S_{b} \rho_{b 0} d x_{1},
$$

где $V_{b}$ - объем воздуха, вытесняемый из штоковой полости; $x_{10}, \rho_{b 0}-$ исходная позиция поршня и плотность воздуха в исходной позиции. В соответствии с уравнением Менделеева - Клапейрона запишем

$$
d \rho_{b}=\frac{d p_{b}}{R T} \text { и } d m_{b}=\frac{S_{b} x_{10} d \rho_{b}}{R T_{b}}+S_{b} \rho_{b 0} d x_{1} .
$$

Первое слагаемое этого уравнения соответствует массе воздуха, оставшейся в полости пневмоцилиндра вследствие изменившейся плотности, 
второе слагаемое - массе воздуха, покидающего полость пневмоцилиндра при исходной плотности.

Масса воздуха, выходящая из полости $S_{b}$ через отводящее устройство за время $d t$,

$$
d m_{b}=G_{b} d t=Z_{\text {вх }} k_{\beta b} P_{b} d t
$$

Для определения параметра истечения $k_{\beta b}$ необходимо оценить значение показателя критичности $\beta_{b}=\left(p_{b}-\Delta p_{b}\right) / p_{b}$, где $\Delta p_{b}$ - потеря давления в отводящем устройстве. Давление в штоковой полости определим из уравнения (6): $p_{b}=\left(p_{a} S_{a}+k_{1} x_{1}\right) / S_{b}$. Очевидно, что масса воздуха, выходящая через отводящее устройство пневмоцилиндра ПЦ1, равна второму слагаемому уравнения (13):

$$
Z_{\mathrm{Bx}} k_{\beta b}\left(p_{a} S_{a}+k_{1} x_{1}\right) d t=S_{b}^{2} \rho_{b 0} d x_{1} .
$$

2. Заполнение поршневой полости $\boldsymbol{S}_{\boldsymbol{a}}$. Масса воздуха, заполняющая высвобождаемый объем в полости $S_{a}$,

$$
d m_{2}=d\left(V_{a} \rho_{a}\right)=S_{a} d\left(x_{1} \rho_{a}\right)=S_{a} x_{10} d \rho_{a}=S_{a} \rho_{a 0} d x_{1} .
$$

В соответствии с уравнением Менделеева — Клапейрона имеем

$$
d \rho_{a}=\frac{d p_{a}}{R T_{a}} \text { и } d m_{a}=\frac{S_{a} x_{10} d p_{a}}{R T_{a}}+S_{a} \rho_{a 0} d x_{1}
$$

Первое слагаемое этого уравнения соответствует массе воздуха, оставшейся в полости пневмоцилиндра вследствие изменившейся плотности, второе слагаемое - массе воздуха, которая должна поступить в полость пневмоцилиндра при исходной плотности.

Масса воздуха, поступающая в полость $S_{a}$ через подводящее устройство за время $d t$, представляет собой сумму массы воздуха, вытесняемого из штоковой полости пневмоцилиндра ПЦ2, и массы воздуха, проходящей через обратный клапан $K_{a}$, при этом на входе в подводящее устройство установится давление подпитки $p_{0}$ :

$$
d m_{a}=G_{a} d t=Z_{\text {вых }} k_{\beta b} p_{0} d t .
$$

Параметр истечения $k_{\beta a}$ оценивается показателем критичности $\beta_{a}=p_{a} / \rho_{0}$. Масса воздуха, поступающая в поршневую полость пневмоцилиндра ПЦ1 через подводящее устройство, равна второму слагаемому уравнения (14) 


$$
Z_{\text {вых }} k_{\beta a} p_{0} d t=S_{a} \rho_{a 0} d x_{1} .
$$

\section{3. Опорожнение штоковой полости $\boldsymbol{S}_{d}$ пневмоцилиндра ПЦ2.} Масса воздуха, вытесняемая поршнем из полости $S_{d}$,

$$
d m_{d}=d\left(V_{d} \rho_{d}\right)=S_{d} d\left(x_{2} \rho_{d}\right)=S_{d} x_{20} d \rho_{d}+S_{d} \rho_{d 0} d x_{2} .
$$

$\mathrm{B}$ соответствии с уравнением Менделеева — Клапейрона $d \rho_{d}=d p_{d} / R T_{d}$ и $d m_{d}=\frac{S_{d} x_{20} d p_{d}}{R T_{d}}+S_{d} \rho_{d 0} d x_{2}$.

Масса воздуха, выходящая из полости $S_{d}$ через отводящее устройство за время $d t$,

$$
d m_{d}=G_{d} d t=Z_{\mathrm{BX}} k_{\beta d} p_{d} d t .
$$

Параметр истечения $k_{\beta d}$ оценивается через показатель критичности $\beta_{d}=\left(p_{d}-\Delta p_{d}\right) / p_{d}$, где $\Delta p_{d}$ - потеря давления в отводящем устройстве. Давление $p_{d}$ в штоковой полости выразим из (8):

$$
p_{d}=\frac{\left(p_{c} S_{c}-k_{2} x_{2}\right)}{S_{d}}
$$

Масса воздуха, выходящая через отводящее устройство, равна второму слагаемому уравнения (15)

$$
Z_{\mathrm{BX}} k_{\beta d}\left(p_{c} S_{c}-k_{2} x_{2}\right) d t=S_{d}^{2} \rho_{d 0} d x_{2} .
$$

4. Заполнение поршневой полости $S_{c}$ пневмоцилиндра ПЦ2. Масса воздуха, заполняющая высвобождаемый объем,

$$
d m_{c}=d\left(V_{c} \rho_{c}\right)=S_{c} d\left(x_{2} \rho_{c}\right)=S_{c} x_{20} d \rho_{c}+S_{c} \rho_{c 0} d x_{2} .
$$

В соответствии с уравнением Менделеева - Клапейрона запишем

$$
d \rho_{c}=\frac{d p_{c}}{R T_{c}} \text { и } d m_{c}=\frac{S_{c} x_{20} d p_{c}}{R T_{c}}+S_{c} \rho_{c 0} d x_{2} .
$$

Масса воздуха, поступающая в полость $S_{c}$ через подводящее устройство пневмоцилиндра ПЦ2, 


$$
d m_{c}=G_{c} d t=Z_{\text {вых }} k_{\beta c}\left(p_{c}+\Delta p_{c}\right) d t .
$$

Параметр истечения $k_{\beta c}$ оценивается показателем критичности $\beta_{c}=$ $=p_{c} /\left(p_{c}+\Delta p_{c}\right)$, где $\Delta p_{c}$ - потеря давления в подводящем устройстве. Давление в поршневой полости выразим из уравнения (8):

$$
p_{c}=\frac{\left(p_{d} S_{d}-k_{2} x_{2}\right)}{S_{c}} .
$$

Масса воздуха, поступающая в поршневую полость через подводящее устройство, равна второму слагаемому уравнения (16)

$$
Z_{\text {вых }} k_{\beta c}\left(p_{d} S_{d}+k_{2} x_{2}+\Delta p_{c} S_{c}\right) d t=S_{c}^{2} \rho_{c 0} d x_{2}
$$

Таким образом, динамика копирующего пневмопривода на прямом ходе задающего пневмоцилиндра описывается системой уравнений, полученных в условиях тепломассообмена:

$$
\begin{gathered}
Z_{\text {вых }} k_{\beta a} p_{0} d t=S_{a} \rho_{a 0} d x_{1} ; \\
Z_{\text {вх }} k_{\beta d}\left(p_{c} S_{c}-k_{2} x_{2}\right) d t=S_{d}^{2} \rho_{d 0} d x_{2} ; \\
Z_{\text {вх }} k_{\beta d}\left(p_{a} S_{a}+k_{1} x_{1}\right) d t=S_{b}^{2} \rho_{b 0} d x_{1} ; \\
Z_{\text {вых }} k_{\beta c}\left(p_{d} S_{d}+k_{2} x_{2}+\Delta p_{c} S_{c}\right) d t=S_{c}^{2} \rho_{c 0} d x_{2} ; \\
p_{b}=\frac{\left(p_{a} S_{a}+k_{1} x_{1}\right)}{S_{b}} ; \\
p_{d}=\frac{\left(p_{c} S_{c}-k_{2} x_{2}\right)}{S_{d}} .
\end{gathered}
$$

Обратный ход поршня пневмоцилиндра ПЦ1. Запишем выражения массовых расходов в процессах заполнения и опорожнения полостей пневмоцилиндров ПЦ1 и ПЦ2 при обратном ходе поршня пневмоцилиндра ПЦ1. 
1. Опорожнение поршневой полости $\boldsymbol{S}_{\boldsymbol{a}}$ пневмоцилиндра ПЦ1.

Масса воздуха, вытесняемая поршнем из полости $S_{a}$,

$$
d m_{a}=d\left(V_{a} \rho_{a}\right)=S_{a} d\left(x_{1} \rho_{a}\right)=S_{a} x_{10} \frac{d p_{a}}{R T_{a}}+S_{a} \rho_{a 0} d x_{1} .
$$

Масса воздуха, выходящая из полости $S_{a}$ через отводящее устройство,

$$
d m_{a}=G_{a} d t=Z_{\mathrm{Bx}} k_{\beta a} p_{a} d t
$$

Показатель критичности $\beta_{a}=\left(p_{a}-\Delta p_{a}\right) / p_{a}$. Давление в полости $S_{a}$ $p_{a}=\left(p_{b} S_{b}-k_{1} x_{1}\right) / S_{a}$.

Уравнение баланса масс

$$
Z_{\mathrm{BX}} k_{\beta a}\left(p_{b} S_{b}-k_{1} x_{1}\right) d t=S_{a}^{2} \rho_{a 0} d x_{1} .
$$

2. Заполнение штоковой полости $S_{b}$ пневмоцилиндра ПЦ1. Масса воздуха, заполняющего полость $S_{b}$,

$$
d m_{b}=d\left(V_{b} \rho_{b}\right)=S_{b} d\left(x_{1} \rho_{b}\right)=S_{b} x_{10} \frac{d p_{b}}{R T_{b}}+S_{b} \rho_{b 0} d x_{1}
$$

Масса воздуха, поступающего в полость $S_{b}$ через подводящее устройство, будет определяться как разность массы воздуха, вытесняемого поршнем из полости $S_{c}$ пневмоцилиндра ПЦ2, и массы воздуха, проходящей через клапан подпора $K_{b}$, при этом на входе в полость установится давление $p_{b}{ }^{\prime}$, характеризуемое настройкой клапана:

$$
d m_{b}=G_{b} d t=Z_{\text {вых }} k_{\beta b} p_{b} d t .
$$

Показатель критичности $\beta_{b}=p_{b} / p_{0}$, давление в полости $S_{b} p_{b}=$ $=\left(p_{a} S_{a}+k_{1} x_{1}\right) / S_{b}$.

3. Опорожнение поршневой полости $\boldsymbol{S}_{\boldsymbol{c}}$ пневмоцилиндра ПЦ2. Масса воздуха, вытесняемая поршнем из полости $S$,

$$
d m_{c}=d\left(V_{c} \rho_{c}\right)=S_{c} d\left(x_{2} \rho_{c}\right)=\frac{S_{c} x_{20} d p_{c}}{R T_{c}}+S_{c} \rho_{c 0} d x_{2}
$$


Масса воздуха, выходящего из полости $S_{c}$ через отводящее устройство,

$$
d m_{c}=G_{c} d t=Z_{\mathrm{Bх}} k_{\beta c} p_{c} d .
$$

Показатель критичности $\beta_{c}=\left(p_{c}-\Delta p_{c}\right) / p_{c}$, давление в полости $S_{c} p_{c}=$ $=\left(p_{d} S_{d}+p_{d} S_{d}\right) / S_{c}$.

4. Заполнение штоковой полости $\boldsymbol{S}_{d}$ пневмоцилиндра ПЦ2. Масса воздуха, заполняющего полость $S_{d}$,

$$
d m_{d}=d\left(V_{d} \rho_{d}\right)=S_{d} d\left(x_{2} \rho_{d}\right)=\frac{S_{d} x_{20} d p_{d}}{R T_{d}}+S_{d} \rho_{d 0} d x_{2} .
$$

Масса воздуха, поступающая в полость $S_{d}$ через подводящее устройство, будет вычисляться как разность массы воздуха, вытесняемого из полости $S_{a}$ пневмоцилиндра ПЦ1, и массы воздуха, выходящего через клапан подпора $K_{d}$; при этом на входе в полость установится давление $p_{d}{ }^{\prime}$, определяемое настройкой клапана:

$$
d m_{d}=G_{d} d t=Z_{\mathrm{B \textrm { }}} k_{\beta d} p_{d} d t .
$$

Показатель критичности $\beta_{d}=p_{d} / p_{0}^{\prime}$, давление в полости $S_{d} p_{d}=$ $=\left(p_{c} S_{c}-k_{2} x_{2}\right) / S_{d}$.

Динамика копирующего пневмопривода на обратном ходе задающего пневмоцилиндра описывается системой уравнений (20)-(28). Исследования копирующего пневмопривода, выполненного на базе одинаковых пневмоцилиндров с параметрами 30; 16; 150 мм при давлении подпитки $p_{0}=0,2$ МПа и давлении настройки клапанов подпора $p_{K}=0,22$ МПа подтвердили адекватность разработанной математической модели.

\section{ЛИТЕРАТУРА}

[1] Дмитриев В.Н., Градецкий В.Г. Основы пневмоавтоматики. Москва, Машиностроение, 1973,360 с.

[2] Нагорный В.С., Денисов А.А. Устройства автоматики гидро- и пневмосистем. Москва, Высшая школа, 1991, 367 с.

[3] Герц Е.В. Динамика пневматических систем машин. Москва, Машиностроение, 1985, 255 с.

[4] Башта Т.М., Руднев С.С., Некрасов Б.Б. Гидравлика, гидромашины и гидроприводы. Москва, Машиностроение, 1982, 423 с.

[5] Башта Т.М. Гидропривод и гидропневмоавтоматика. Москва, Машиностроение, 1972, 320 с. 
[6] Пильгунов В.Н. Высокоточный широкодиапазонный расходомер сжатого воздуха - измеритель малых перемещений. Электронное научно-техническое издание «Наука и образование», № 8, 2012. URL: http://technomag.edu. ru. doi: 10.7463/0812.0451962 (дата обращения 20.05.2013).

Статья поступила в редакцию 08.06.2013

Ссылку на эту статью просим оформлять следующим образом:

Пильгунов В.Н., Ефремова К.Д. Копирующий пневмопривод. Инженерный журнал: наука и инновации, 2013, вып. 4. URL: http:/engjournal.ru/catalog/machin/ hydro/686.html

Ефремова Клара Дмитриевна - канд. техн. наук, доцент кафедры «Гидромеханика, гидромашины и гидропневмоавтоматика» МГТУ им. Н.Э. Баумана. Автор более 70 научных и методических работ в области пневмоавтоматики и механики жидкости и газа. e-mail: kde@bmstu.ru

Пильгунов Владимир Николаевич родился в 1941 г., окончил МВТУ им. Н.Э. Баумана в 1964 г. Канд. техн. наук, доцент кафедры «Гидромеханика, гидравлические машины и гидропневмоавтоматика» МГТУ им. Н.Э. Баумана. Автор более 60 научных работ в области механики жидкости и гидропневмоавтоматики. e-mail: vnp41@yandex.ru 